\title{
Progress in the search for neuronal mechanisms coupling type 2 diabetes to obesity
}

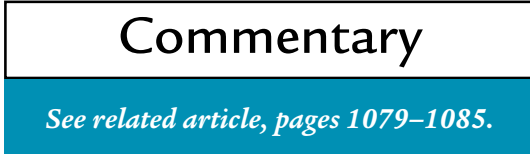

\author{
Michael W. Schwartz \\ Division of Metabolism, Endocrinology and Nutrition, University of Washington, Harborview Medical Center, \\ 325 Ninth Avenue, Box 359757, Seattle, Washington 98104-2499, USA. \\ Phone: (206) 341-5288; Fax: (206) 341-5293; E-mail: mschwart@u.washington.edu.
}

J. Clin. Invest. 108:963-964 (2001). DOI:10.1172/JCI200114127.

Obesity, insulin resistance, and type 2 diabetes are a tightly linked and increasingly common triad of metabolic disorders. A well-supported and widely accepted explanation for their association is that obesity-induced insulin resistance in tissues such as muscle, liver, and fat increases the demand for insulin, and that type 2 diabetes ensues when this heightened demand cannot be met by defective pancreatic $\beta$ cells. Despite the undeniable importance of adipocytes in the regulation of insulin sensitivity (see refs. 1-3 for recent reviews and insights into this interaction), this understanding of the pathogenesis of type 2 diabetes may yet be incomplete. Growing evidence suggests that the CNS plays a key role in glucose homeostasis, via brain pathways that overlap with those controlling food intake and body weight. Advancing this notion a step further is the study by Obici et al., published in this issue of the $J C I$ (4), implicating a specific hypothalamic neuronal pathway - the melanocortin pathway - in the control of insulin sensitivity in peripheral tissues. The recognition that neuronal melanocortin signaling also figures prominently in energy homeostasis, the process whereby energy intake is matched to energy expenditure over time, suggests an intimate coupling of neuronal mechanisms regulating body weight and glucose metabolism (Figure 1). Disorders affecting key neuronal pathways can therefore be included among potential mechanisms linking obesity to type 2 diabetes (5).

\section{Neuroendocrine control of energy homeostasis}

The hypothesis that humoral signals generated in proportion to body fat stores are critical for energy homeostasis gained wide acceptance following the demonstration that genetic deficiency of the adipocyte hormone, leptin, causes severe hyperphagia and obesity (6) that is reversed by leptin administration ( 7 , 8). The subsequent identification of discrete, leptin-responsive hypothalamic pathways clearly established that circu- lating signals generated in proportion to body adiposity provide afferent input to brain areas involved in energy homeostasis (9). Although leptin likely plays a more critical role, insulin also provides afferent input to the CNS regarding the sufficiency of body fat stores (5). Both hormones circulate at levels proportionate to body adiposity and enter the brain, possibly via receptor-mediated transport across blood-brain barrier endothelium. Receptors for insulin and for leptin are concentrated in key hypothalamic areas such as the arcuate nucleus, and intracerebroventricular (icv) infusion of low doses of either hormone reduces food intake and body weight (reviewed in refs. 5 , 9). Energy restriction, which lowers insulin and leptin levels, is therefore proposed to activate an integrated set of CNS responses that limit weight loss and promote the recovery of depleted fat stores when sufficient food becomes available $(5,9,10)$.

While a sizable number of neuronal systems are now implicated in energy homeostasis, none is more crucial than a

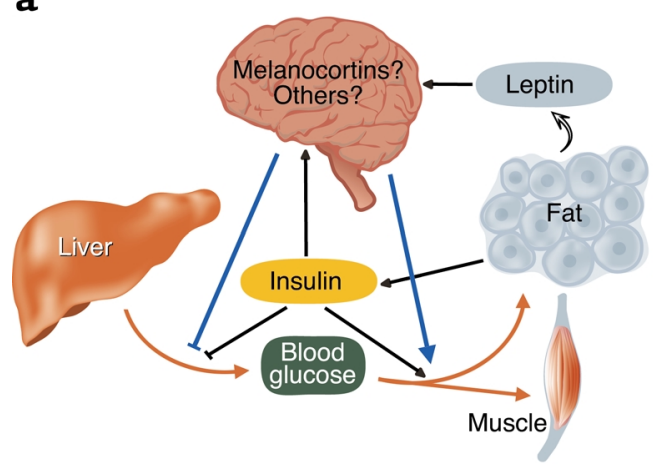

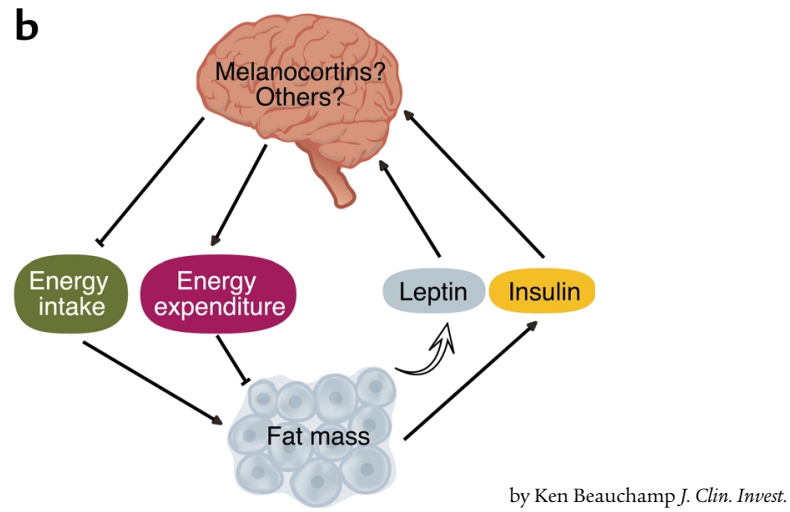

Figure 1

CNS regulation of glucose metabolism. (a) Neuronal pathways including the melanocortin system potentiate insulin's ability to inhibit hepatic glucose production and to increase glucose uptake into muscle and fat. Insulin and leptin, which circulate at levels proportionate to body adiposity, activate neuronal glucose-lowering pathways. Since the same neuroendocrine control system is critical for normal energy homeostasis (b), defects in its signaling can induce both insulin resistance and obesity via distinct but complementary mechanisms and thus predispose to type 2 diabetes. 
the hypothalamic melanocortin pathway. Melanocortins are peptides such as $\alpha$-melanocyte-stimulating hormone $(\alpha-\mathrm{MSH})$ that are cleaved from the proopiomelanocortin (POMC) precursor. POMC-synthesizing neurons within the arcuate nucleus are stimulated by leptin (11) and can reduce food intake and increase energy expenditure by activating two neuronal melanocortin receptor subtypes: $\mathrm{Mc} 3 \mathrm{r}$ and, still more dramatically, Mc4r. The weight-reducing actions of melanocortins are opposed by an adjacent subset of arcuate nucleus neurons that contain both agouti-related peptide (AgRP), an endogenous $\mathrm{Mc} 3 / 4 \mathrm{r}$ antagonist, and neuropeptide $\mathrm{Y}$ (NPY, a potent stimulator of food intake). Unlike POMC neurons, NPY/AgRP neurons can be inhibited by leptin or insulin. Thus, as weight loss lowers insulin and leptin levels, NPY/AgRP neurons are activated, POMC neurons are inhibited $(9,10)$, and body fat begins to accumulate once nutrient availability permits. That this neuroendocrine control system is essential for normal energy homeostasis is confirmed by the obese phenotype of mice with loss-of-function mutations affecting any of several key signaling molecules (reviewed in ref. 12), including leptin $(o b / o b)$, leptin receptor $(d b / d b)$, POMC, Mc4r, and neuronal insulin receptors (13). Importantly, striking parallels exist between humans and mice with respect to the obesityinducing consequences of mutations affecting neuronal leptin or melanocortin signaling (12).

\section{CNS control of glucose \\ homeostasis}

Several observations suggest that leptin deficiency causes hyperglycemia and hyperinsulinemia via mechanisms independent of obesity per se. For example, leptin deficiency contributes to the syndrome of severe insulin resistance and diabetes characteristic of congenital absence of adipose tissue (lipodystrophy) in mice $(3,14)$, and leptin potently lowers insulin and glucose levels in $o b / o b$ mice independent of its effects on food intake and body weight (15). Evidence that leptin infusion directly into the hypothalamus potentiates insulin action in muscle and fat (16) suggests that its effects on glucose homeostasis involve a central site of action. Centrally administered insulin also promotes insulin-stimulated glucose utilization in peripheral tissues, whereas blockade of hypothalamic insulin signaling (17) or neuron-specific deletion of insulin receptors (13) causes systemic insulin resistance. Intact hypothalamic signaling by insulin and leptin therefore appears to be obligatory not only for normal energy homeostasis, but for normal glucose homeostasis as well.

To investigate whether the same is true for hypothalamic melanocortin signaling, Obici and colleagues (4) first determined the effect of icv infusion of $\alpha-\mathrm{MSH}$ on peripheral tissue insulin sensitivity in rats using the euglycemic, hyperinsulinemic clamp method. Following a week-long icv infusion of $\alpha$ MSH at a dose below that needed to reduce food intake, both insulin-stimulated glucose disposal and insulininduced suppression of hepatic glucose production were increased. Increased neuronal melanocortin receptor signaling is therefore sufficient to increase insulin sensitivity in vivo, although it remains to be determined whether this effect occurs independently of melanocortin-induced decreases of body adiposity. The more compelling question of whether neuronal melanocortin receptor signaling is required for normal glucose homeostasis was addressed by icv infusion of the melanocortin $3 / 4$ receptor antagonist, SHU9119. The finding that insulin resistance results from pharmacological blockade of neuronal melanocortin receptors, even when changes in body adiposity are prevented by controlling food intake (4), suggests that intact neuronal melanocortin signaling is required for normal insulin sensitivity in peripheral tissues, independent of its effects on energy balance (Figure 1). This concept is further supported by evidence that hyperinsulinemia and insulin resistance develop at an early age in Mc4r-deficient mice, prior to the onset of hyperphagia and obesity (18).

\section{A role for the brain in the pathogenesis of both obesity and type 2 diabetes?}

These observations raise a number of important questions. What efferent mechanisms transduce the hypothalamic actions of insulin, leptin, and melanocortins into altered insulin sensitivity in liver and muscle? Is the melanocortin system downstream of neuronal leptin and insulin signaling in the control of glucose homeostasis, or does it play an important role independent of input from hormonal stimuli (or both)? Finally, to what extent might neuronal defects contribute to the pathogenesis of both obesity and type 2 diabetes? Consider that in addition to causing insulin resistance in liver, fat, and muscle (1), obesity also causes leptin resistance in the hypothalamus (19). Defective signaling within key neuronal pathways that control both energy homeostasis and glucose homeostasis may therefore contribute to the link between type 2 diabetes and obesity.

1. Kahn, B.B., and Flier, J.S. 2000. Obesity and insulin resistance. J. Clin. Invest. 106:473-481.

2. Steppan, C.M., et al. 2001. The hormone resistin links obesity to diabetes. Nature. 409:307-312

3. Yamauchi, T., et al. 2001. The fat-derived hormone adiponectin reverses insulin resistance associated with both lipoatrophy and obesity. Nat. Med. 7:941-946

4. Obici, S., et al. 2001. Central melanocortin receptors regulate insulin action. J. Clin. Invest. 108:1079-1085.

5. Porte, D., Jr., et al. 1998. Obesity, diabetes and the central nervous system. Diabetologia. 41:863-881.

6. Zhang, Y., et al. 1994. Positional cloning of the mouse obese gene and its human homologue. Nature. 372:425-432.

7. Campfield, L.A., Smith, F.J., Guisez, Y., Devos, R. and Burn, P. 1995. Recombinant mouse OB protein: evidence for a peripheral signal linking adiposity and central neural networks. Science. 269:546-549.

8. Weigle, D.S., et al. 1995. Recombinant ob protein reduces feeding and body weight in the ob/ob mouse. J. Clin. Invest. 96:2065-2070.

9. Schwartz, M.W., Woods, S.C., Porte, D., Jr., Seeley, R.J., and Baskin, D.G. 2000. Central nervous system control of food intake. Nature. 404:661-671.

10. Flier, J. 1998. What's in a name? In search of leptin's physiologic role. J. Clin. Endocrinol. Metab. 83:1407-1413.

11. Cowley, M.A., et al. 2001. Leptin activates anorexigenic POMC neurons through a neural network in the arcuate nucleus. Nature. 411:480-484.

12. Barsh, G.S., Farooqi, I.S., and O'Rahilly, S. 2000 Genetics of body-weight regulation. Nature. 404:644-651.

13. Bruning, J.C., et al. 2000. Role of brain insulin receptors in control of body weight and reproduction. Science. 289:2122-2125.

14. Shimomura, I., Hammer, R.E., Ikemoto, S., Brown, M.S., and Goldstein, J. 1999. Leptin reverses insulin resistance and diabetes mellitus in mice with congenital lipodystrophy. Nature. 401:73-76.

15. Schwartz, M.W., et al. 1996. Specificity of leptin action on elevated blood glucose levels and hypothalamic neuropeptide $Y$ gene expression in ob/ob mice. Diabetes. 45:531-535.

16. Haque, M.S., et al. 1999. Role of the sympathetic nervous system and insulin in enhancing glucose uptake in peripheral tissues after intrahypothalamic injection of leptin in rats. Diabetes. 48:1706-1712.

17. Obici, S., Feng, Z., and Rossetti, L. 2001. Hypothalamic insulin action is required for the inhibitory effects of insulin on hepatic glucose fluxes. Keystone Symposium: Obesity and the Regulation of Energy Homeostasis. Abstract 114. p. 45.

18. Fan, W., et al. 2000. The central melanocortin system can directly regulate serum insulin levels. Endocrinology. 141:3072-3079.

19. El-Haschimi, K., Pierroz, D.D., Hileman, S.M., Bjorbaek, C., and Flier, J.S. 2000. Two defects contribute to hypothalamic leptin resistance in mice with dietinduced obesity. J. Clin. Invest. 105:1827-1832. 